\title{
Psychosocial wellbeing among children and adults with arthrogryposis: a scoping review
}

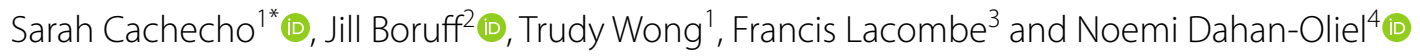

\begin{abstract}
Background: Arthrogryposis multiplex congenita (AMC) is a group of congenital conditions characterized by joint contractures in two or more body areas. Management of AMC starts early in life and focuses on improving mobility and function through intensive rehabilitation and surgical interventions. Psychosocial wellbeing is an important determinant of health and the psychosocial experience of individuals with AMC should be considered in the management of this condition. The aim of this scoping review was to explore what is known about the psychosocial wellbeing of children and adults with AMC, to identify the outcome measures used and to explore the factors associated with psychosocial outcomes in this population.
\end{abstract}

Methods: A comprehensive search in four databases was conducted. Articles discussing psychosocial outcomes and outcome measures used with children or adults with AMC were included. Data on the measures used, psychosocial outcomes, and factors associated with psychosocial outcomes, were extracted and analyzed descriptively and synthesized narratively.

Results: Seventeen articles were included in this scoping review, ten including the pediatric population, six including adults and one article including both children and adults with AMC. The most commonly used outcome measures were the PODCl in the pediatric studies, and the SF-36 in studies on adults. In the pediatric studies, psychosocial outcomes were often secondary, compared to the studies on adults. Results showed that in both children and adults, psychosocial outcomes are comparable with the levels of the general population. Qualitative studies reflected the affective needs of this population and issues with emotional wellbeing. Factors such as fatigue and pain were associated with poorer psychosocial outcomes in adults with an impact on social relationships, intimacy and family planning.

Conclusion: Validated outcome measures, qualitative approaches and longitudinal studies are needed to better understand the psychosocial outcomes in AMC over time. Psychosocial support should be part of the multidisciplinary management of AMC throughout the lifespan.

Keywords: Arthrogryposis multiplex congenita, Psychosocial wellbeing, Quality of life, Outcome measures

${ }^{*}$ Correspondence: scachecho@shrinenet.org

${ }^{1}$ Shriners Hospital for Children-Canada, Montreal, Canada

Full list of author information is available at the end of the article

\section{Background}

Arthrogryposis multiplex congenita (AMC) is a term that describes a group of congenital conditions characterized by joint contractures in two or more body areas [1] and affects 1 in 3000 live births [2, 3]. Joint contractures develop in-utero secondary to a decrease in fetal movement resulting in joint fibrosis and stiffness [4]. original author(s) and the source, provide a link to the Creative Commons licence, and indicate if changes were made. The images or other third party material in this article are included in the article's Creative Commons licence, unless indicated otherwise in a credit line to the material. If material is not included in the article's Creative Commons licence and your intended use is not permitted by statutory regulation or exceeds the permitted use, you will need to obtain permission directly from the copyright holder. To view a copy of this licence, visit http://creativecommons.org/licenses/by/4.0/. The Creative Commons Public Domain Dedication waiver (http://creativeco mmons.org/publicdomain/zero/1.0/) applies to the data made available in this article, unless otherwise stated in a credit line to the data. 
Contractures cause limited joint movement and muscle weakness in the involved body areas, and vary in distribution and severity. While contractures do not progress to previously unaffected joints, they may change over time due to growth and treatment. Depending on the severity and distribution of joint contractures, individuals with AMC may experience limitation in mobility and activities of daily living [5-7]. Other body systems such as the respiratory, gastro-intestinal, and central nervous system may be affected, depending on the underlying diagnosis $[1,3]$. The management of AMC starts early in life with intensive rehabilitation, such as splinting and orthosis, range of motion exercises, strengthening programs, and surgical interventions to correct deformities, all of which aim to improve function $[8-11]$.

The need to develop guidelines for the diagnosis, management and rehabilitation of AMC was identified during the Second International Symposium on Arthrogryposis in St-Petersburg in 2014 [12]. Promoting autonomy in daily activities, quality of life (QOL), and participation and integration in the community were emphasized [12]. In the qualitative study by Elfassy and colleagues, youth with AMC and their parents reported concerns in regards to affective wellbeing [13]. Medical and rehabilitation interventions focus mainly on the physical needs of individuals with AMC while psychosocial wellbeing is an important determinant of health and should not be overlooked [14]. Psychosocial wellbeing is a construct that involves social and emotional wellbeing, and refers to a positive mental state, such as feelings of happiness and satisfaction with life [15]. It includes psychological factors such as mental health, emotional wellbeing, and self-esteem, as well as interpersonal factors or the presence of positive relationships in one's life [16]. Psychosocial wellbeing is one of the components of QOL that needs to be considered in populations with chronic conditions, such as AMC, and factors associated with better or poorer QOL need to be better understood [17]. Individuals with AMC may present varying degrees of physical limitations and may undergo several surgeries throughout their life [18]. Pain has been identified as an important issue and limiting factor in adults with AMC [19]. Therefore, in order to tailor interventions and optimize health and wellbeing throughout the lifespan, it is important to understand the psychosocial experience of individuals with $\mathrm{AMC}$ and to identify influencing factors.

The overall aim of this scoping review was to explore what is known about psychosocial outcomes of children and adults with AMC. Specifically, this scoping review aimed to (1) describe the psychosocial outcomes; (2) identify the outcome measures used to evaluate psychosocial outcomes; and (3) explore the factors that are associated with psychosocial outcomes among children and adults with AMC.

\section{Methods}

A scoping review was selected to map the existing knowledge on the psychosocial outcomes in children and adults with AMC. A scoping review is designed to collect, evaluate and present a comprehensive map of existing evidence on a chosen research topic [20]. Arksey and O'Malley's framework was used in addition to the recommendations by Levac, Colquhoun and O'Brien [21, 22]. The stages of this framework are: (1) identifying the research question, (2) identifying relevant studies, (3) selecting studies, (4) charting the data, and (5) collating, summarizing and reporting the results. An additional optional stage involves consultation with stakeholders. This scoping review was conducted by a team consisting of a young adult with arthrogryposis, clinicians (occupational therapist (OT) and social worker), an information scientist, and a clinician scientist. The research question (stage 1) is described above in the background. The PRISMA Extension for Scoping Reviews was used to verify that all aspects of the scoping review were considered [23].

\section{Identifying and selecting relevant studies}

A health sciences librarian (J.B) developed the search strategy and performed the literature searches in MEDLINE, EMBASE, CINAHL, Cochrane Central, and Proquest Dissertations and Theses from database inception until March 6, 2020, with no limits to the period of time or language restrictions. The MEDLINE strategy was developed with input from the project team. After the initial MEDLINE strategy was finalized, it was adapted for use in the other databases. The search strategy (see Additional file 1) was designed to identify all relevant clinical literature on psychosocial wellbeing in AMC. Results from each database were exported into EndNote and duplicates were removed. Two independent reviewers (S.C and N.D-O) applied the selection criteria (Table 1) for titles and abstracts and then full texts, using the Rayyan software for screening of articles [24]. The two reviewers resolved any conflicts regarding inclusion of articles.

\section{Charting the data}

A data extraction form was created to identify study characteristics and key findings of included studies (i.e., study design, study purpose, sample size, outcome measure, intervention, and results). Two reviewers (S.C and N.D-O) independently piloted the data extraction form on three studies to ensure consistency in data extraction. Data samples were compared between the two reviewers 
Table 1 Selection criteria

\begin{tabular}{lll}
\hline & Inclusion criteria & Exclusion criteria \\
\hline $\begin{array}{l}\text { Population age } \\
\text { Population diagnosis }\end{array}$ & Any age & None \\
Study design & Quantitative, qualitative, mixed methods & $\begin{array}{l}\text { Does not provide separate results for AMC in studies including other } \\
\text { diagnoses }\end{array}$ \\
$\begin{array}{l}\text { Language } \\
\text { Psychosocial outcomes }\end{array}$ & $\begin{array}{l}\text { Expert opinion and review articles } \\
\text { Describes psychosocial outcomes and uses an } \\
\text { outcome measure with a psychosocial compo- } \\
\text { nent }\end{array}$ & $\begin{array}{l}\text { Studies that did not provide English or French translation } \\
\text { Does not describe psychosocial outcomes or use an outcome measure }\end{array}$ \\
\hline
\end{tabular}

and any discrepancies were resolved by discussion. A single reviewer (S.C) then extracted data from included studies and any uncertainties were discussed with the second reviewer. Level of evidence of included studies was assigned by two reviewers (S.C and N.D-O) using the Levels of Evidence for Primary Research guidelines by the Center for Evidence-based medicine [25]. In line with the scoping review methodology and the aims of our project, a critical appraisal and risk of bias assessment of included records was not performed.

\section{Collating, summarizing and reporting the results}

The extracted data was synthesized according to three steps: (1) analyzing the data, (2) reporting the findings, (3) discussing the implications [21]. A descriptive numerical analysis and a narrative synthesis were used to analyze and report findings. The descriptive numerical analysis reflected the nature and distribution of the included studies. The primary units of analysis were the study purposes, the psychosocial outcome measures used and the psychosocial outcomes reported. The factors associated with psychosocial wellbeing from the included studies were synthesized narratively.

\section{Stakeholder consultation}

Stakeholder engagement in research is important to ensure that research is relevant to the target population and that it answers questions that are of importance to the end-users [26]. Consulting with stakeholders may also help identify important themes to consider that are not necessarily reflected in the literature [21]. Exchanges on the elements to consider for the psychosocial wellbeing in this population were held during two virtual meetings in Spring and Summer 2020, with patient representatives (young adult with AMC, parent of a child with AMC and support group representative), clinicians (OT, physiotherapist) and researchers. The discussions that emerged from the stakeholder consultation were summarized and themes were reported inductively.

\section{Results}

\section{Study selection}

The search yielded 512 articles. After duplicates were removed, 354 abstracts were reviewed for study eligibility. Three hundred and nine articles were excluded at screening of titles and abstracts, and 29 articles were excluded at screening of full texts. One publication known to the authors that did not appear in the search was included as well. Therefore, 17 studies were included in the scoping review. Refer to Fig. 1 for PRISMA flowchart.

\section{Study characteristics and level of evidence}

Included studies were published between 1997 and 2020, 15 of which were published after 2010. Studies were published across six different countries, with eight in the United States, five in Canada and the remaining in Europe. All studies were observational studies, specifically, cross-sectional studies $(n=7)$, retrospective cohort studies $(n=7)$, qualitative studies $(n=2)$ and one case series. Studies were classified as Level III $(n=6)$ or Level IV $(n=11)$.

\section{Sample characteristics}

The 17 studies included 718 participants with AMC (range 1-177) ranging from infancy to older adulthood. Six studies included adult populations, 10 included pediatric populations, and one study included both children and adults with AMC. The pediatric studies population age ranged between 13 months to 20 years. Six of the 17 studies specified the underlying diagnosis (e.g., Amyoplasia, distal arthrogryposis) while the other studies identified participants as having "arthrogryposis" or "AMC". One study had participants with different diagnoses but provided separate results for the participant with AMC. Refer to Table 2 for a full list of study and sample characteristics. 
Records identified through database searching

$$
(\mathrm{n}=512)
$$

Additional records identified through other sources

$$
(\mathrm{n}=1)
$$
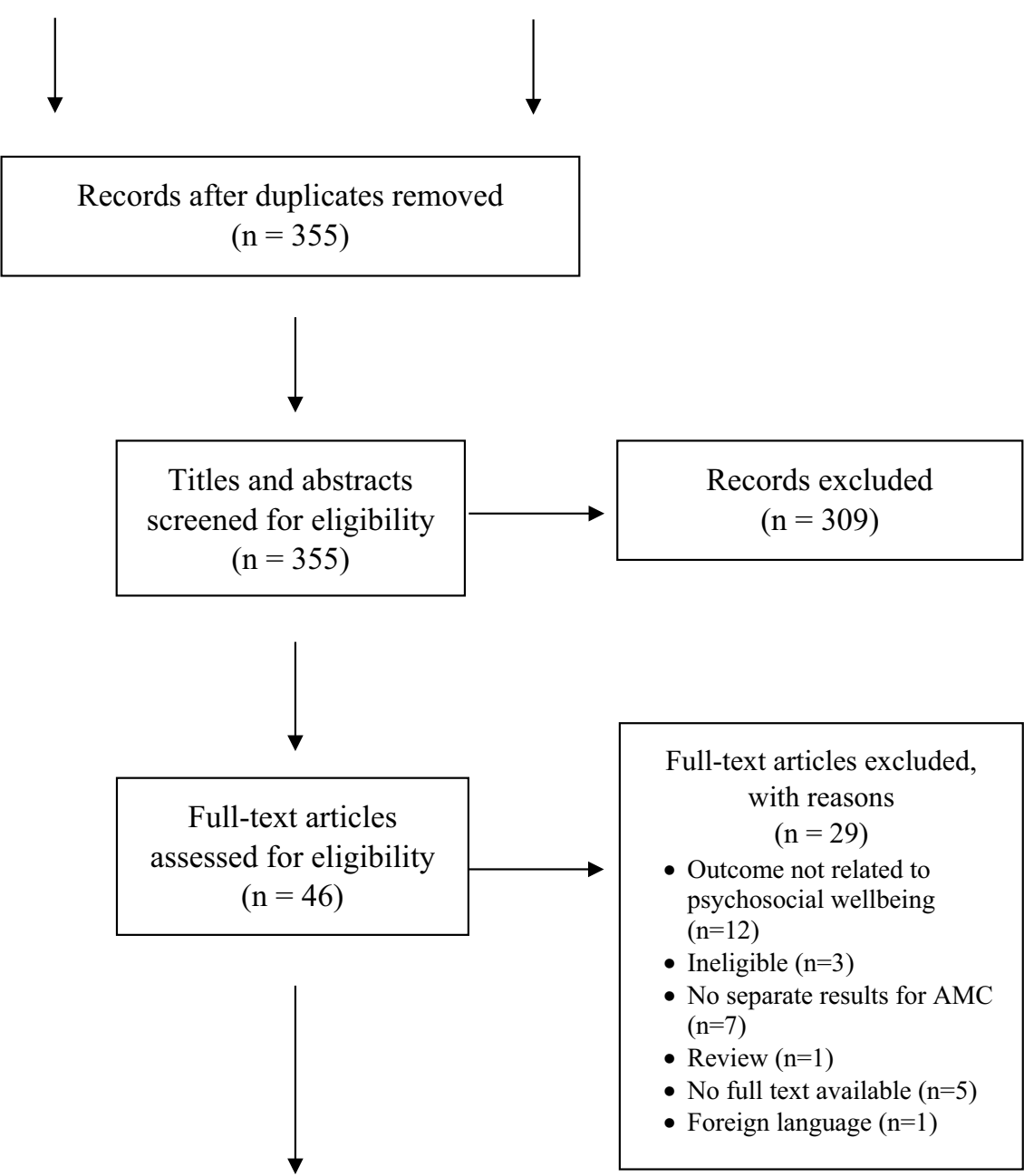

Full-text articles included in the scoping review $(\mathrm{n}=17)$

Fig. 1 PRISMA flowchart

\section{Psychosocial outcome measures}

The outcome measure that was most used in the pediatric studies was the Pediatric Outcome Data Collection instrument (PODCI) [5-7, 27-29]. The PODCI has six domains, one of which measures "happiness with physical condition". All studies using the PODCI were parent-report and two studies also included patient-report among participants older than 11 years $[6,28]$. One study used the Patient-Reported Outcomes Measurement Information System (PROMIS), more specifically the depression, anxiety, and peer relations domains [30]. Other outcome measures with questions 


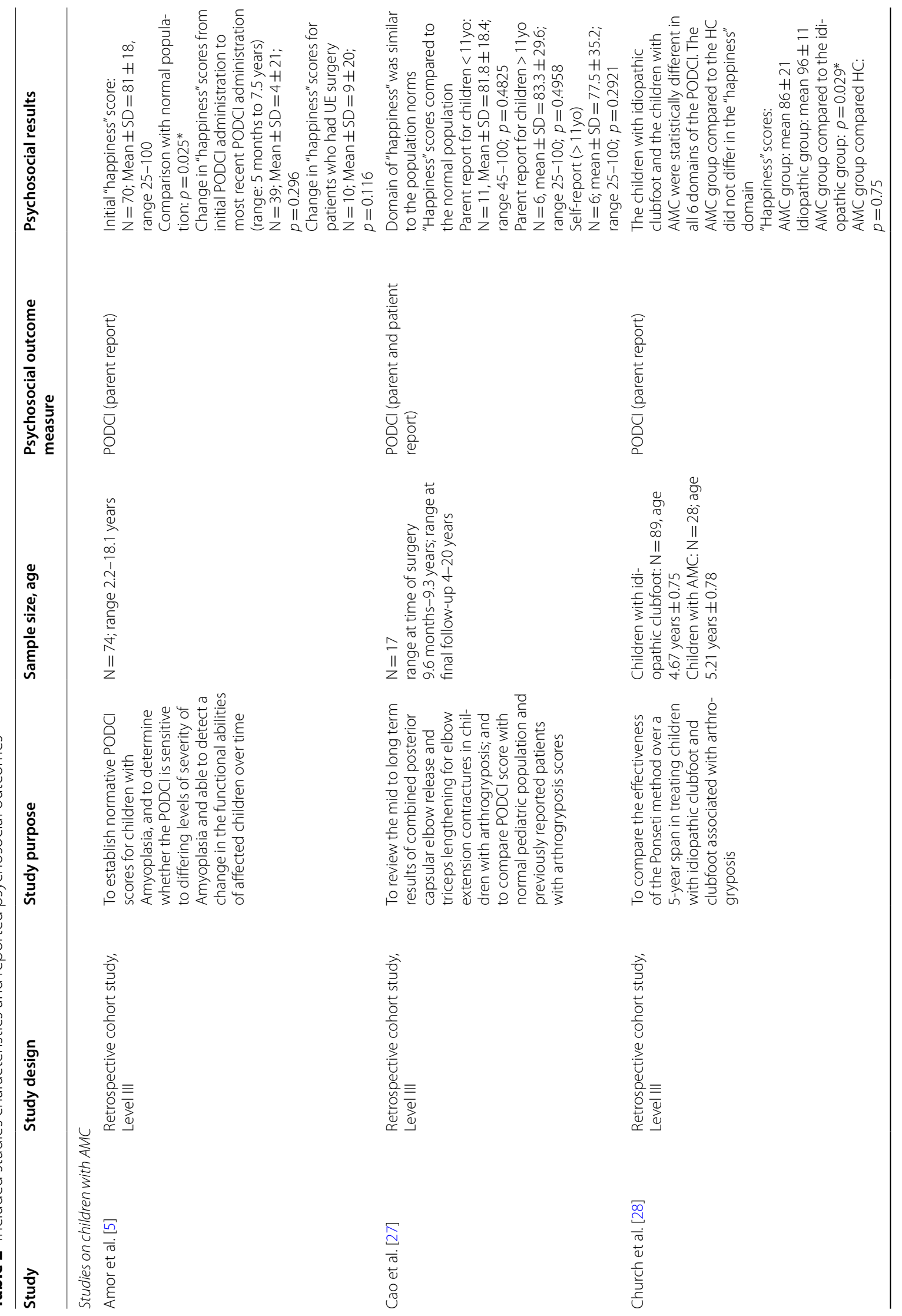




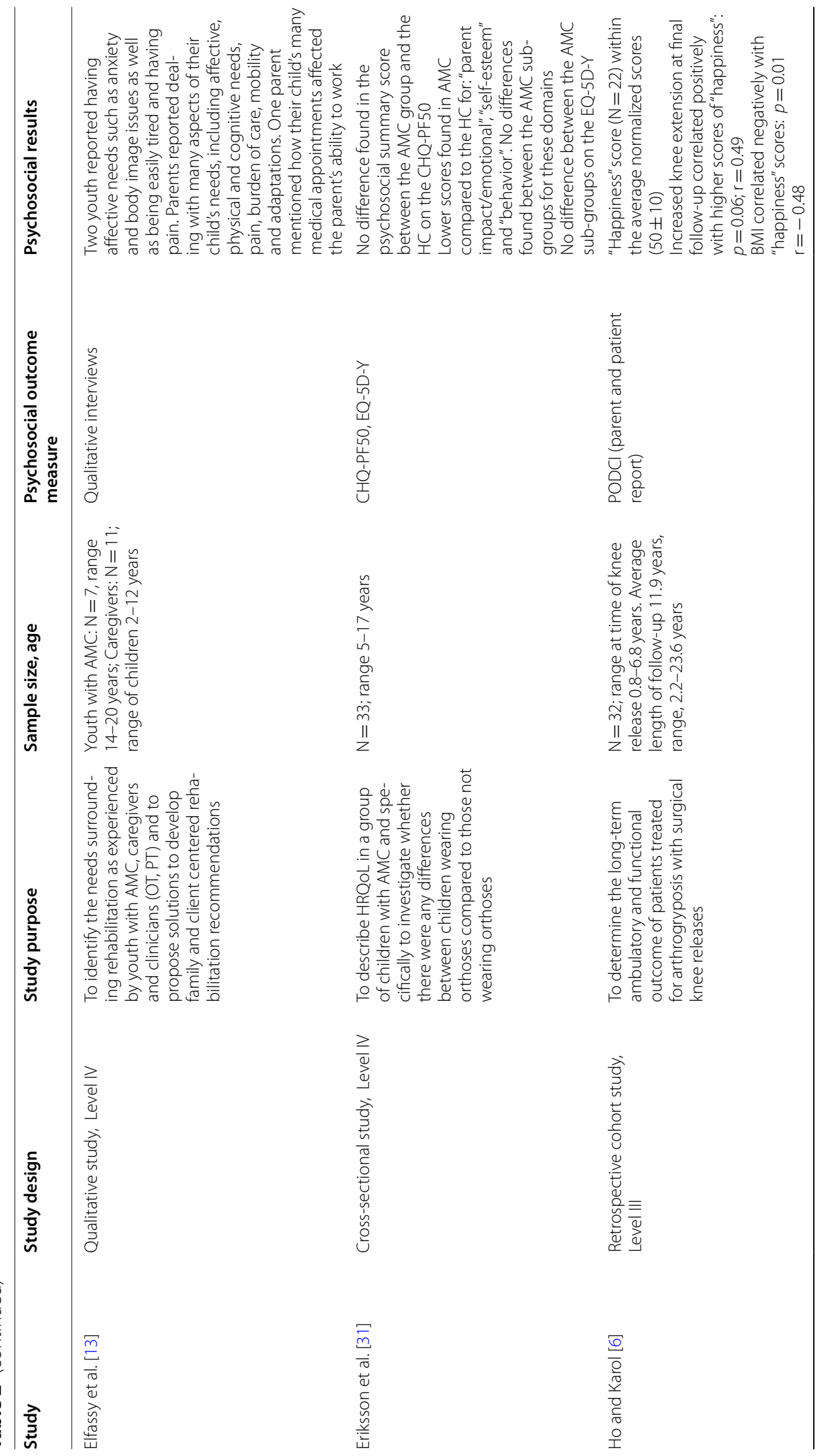




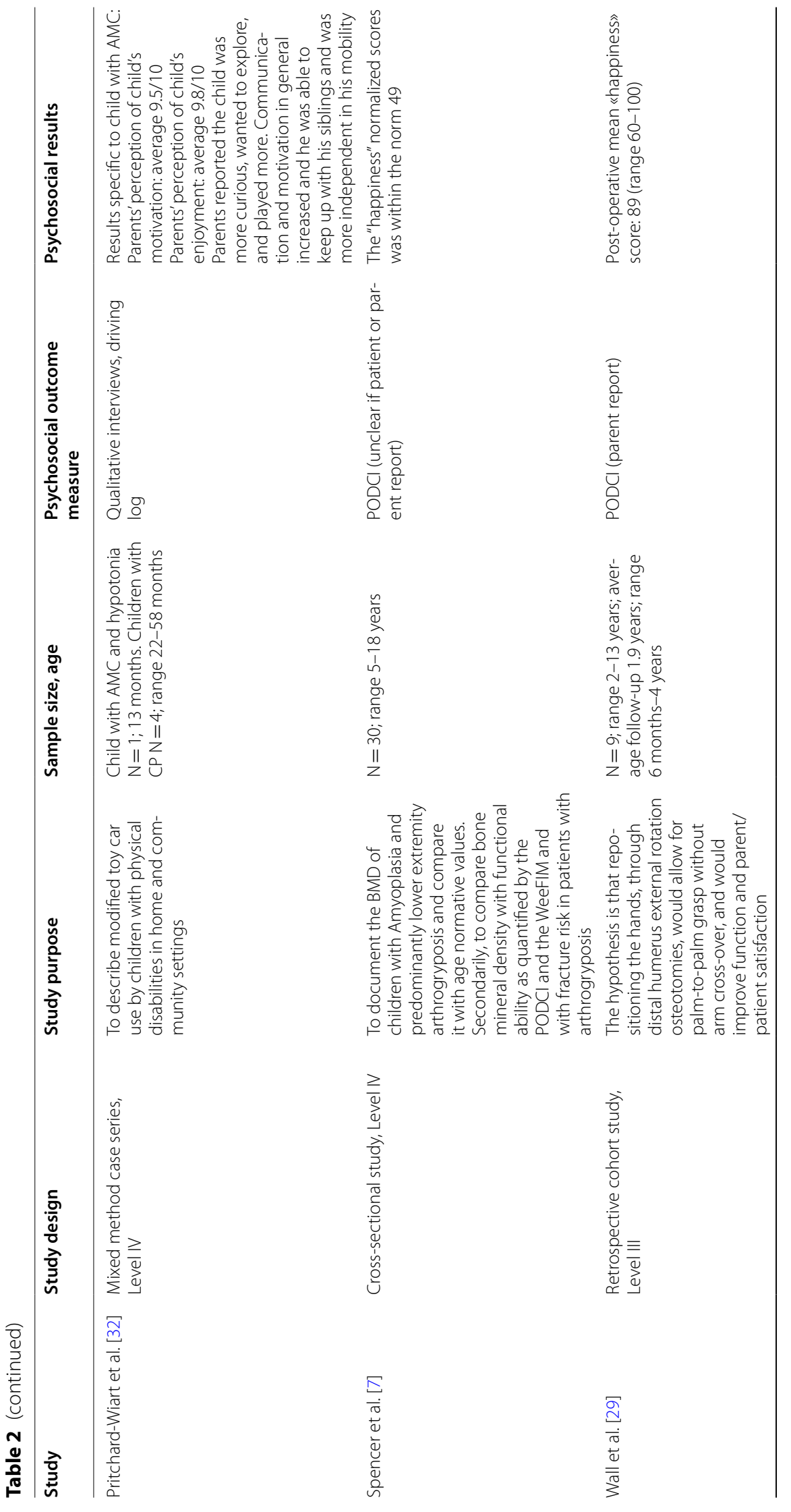




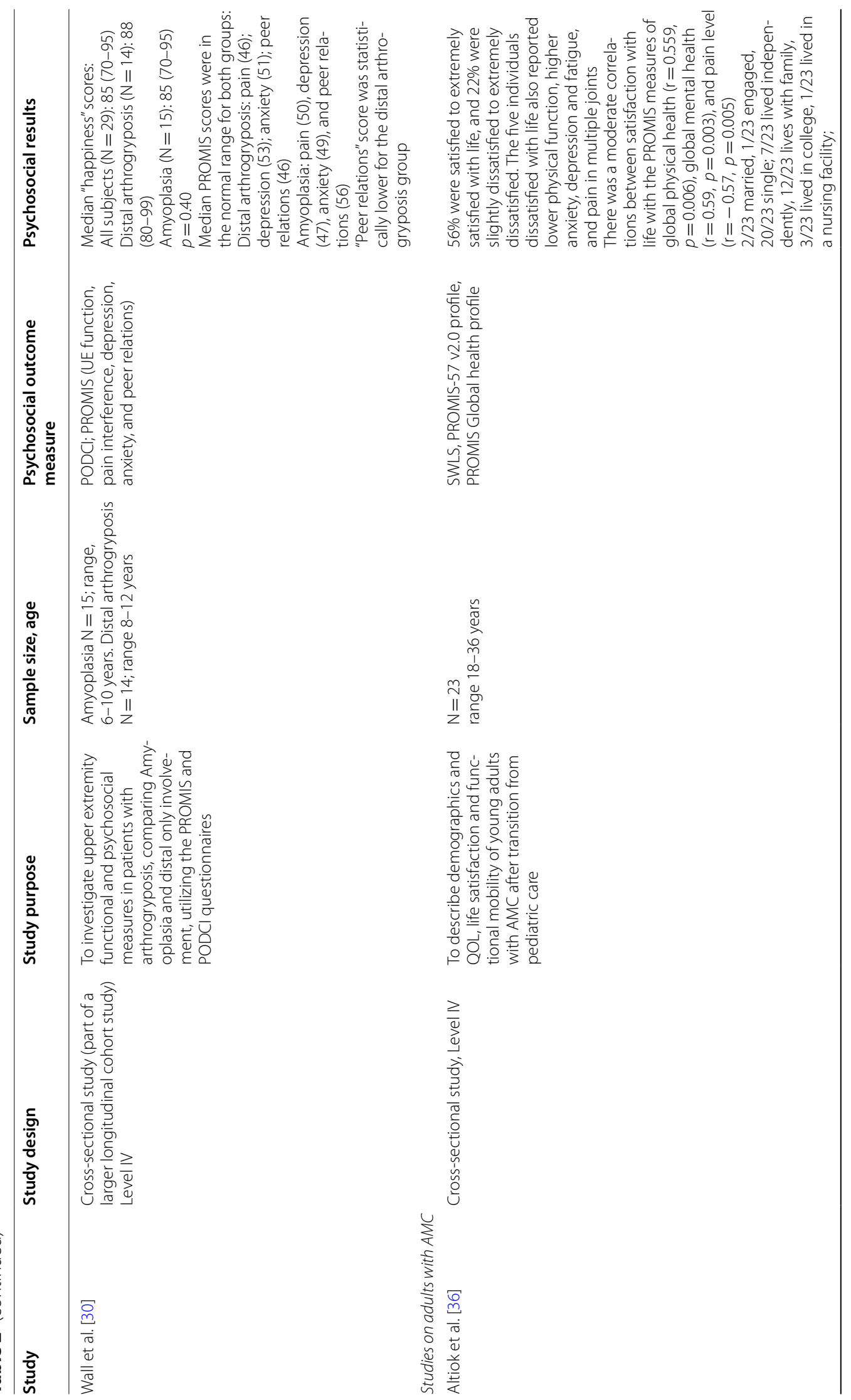




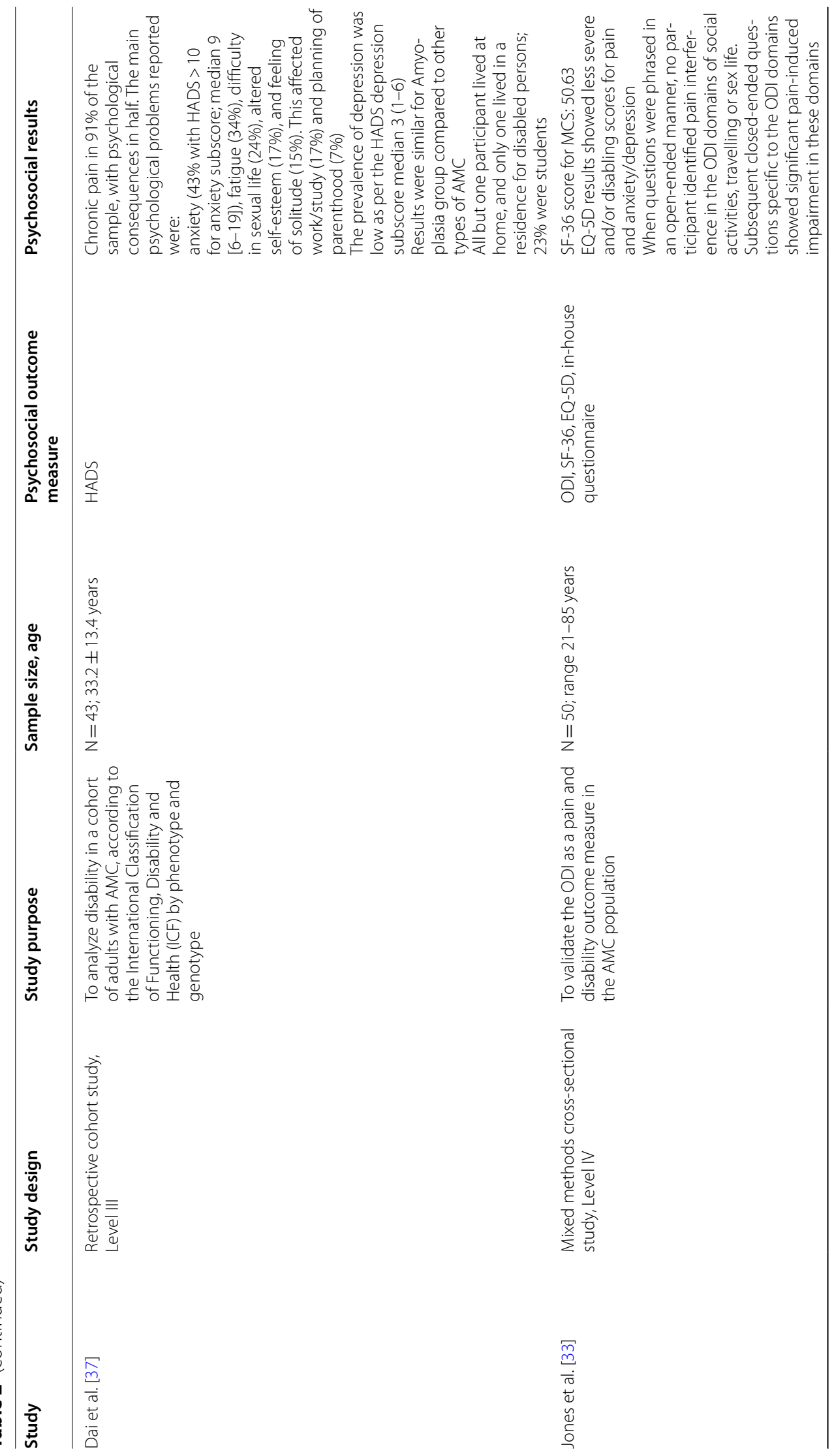




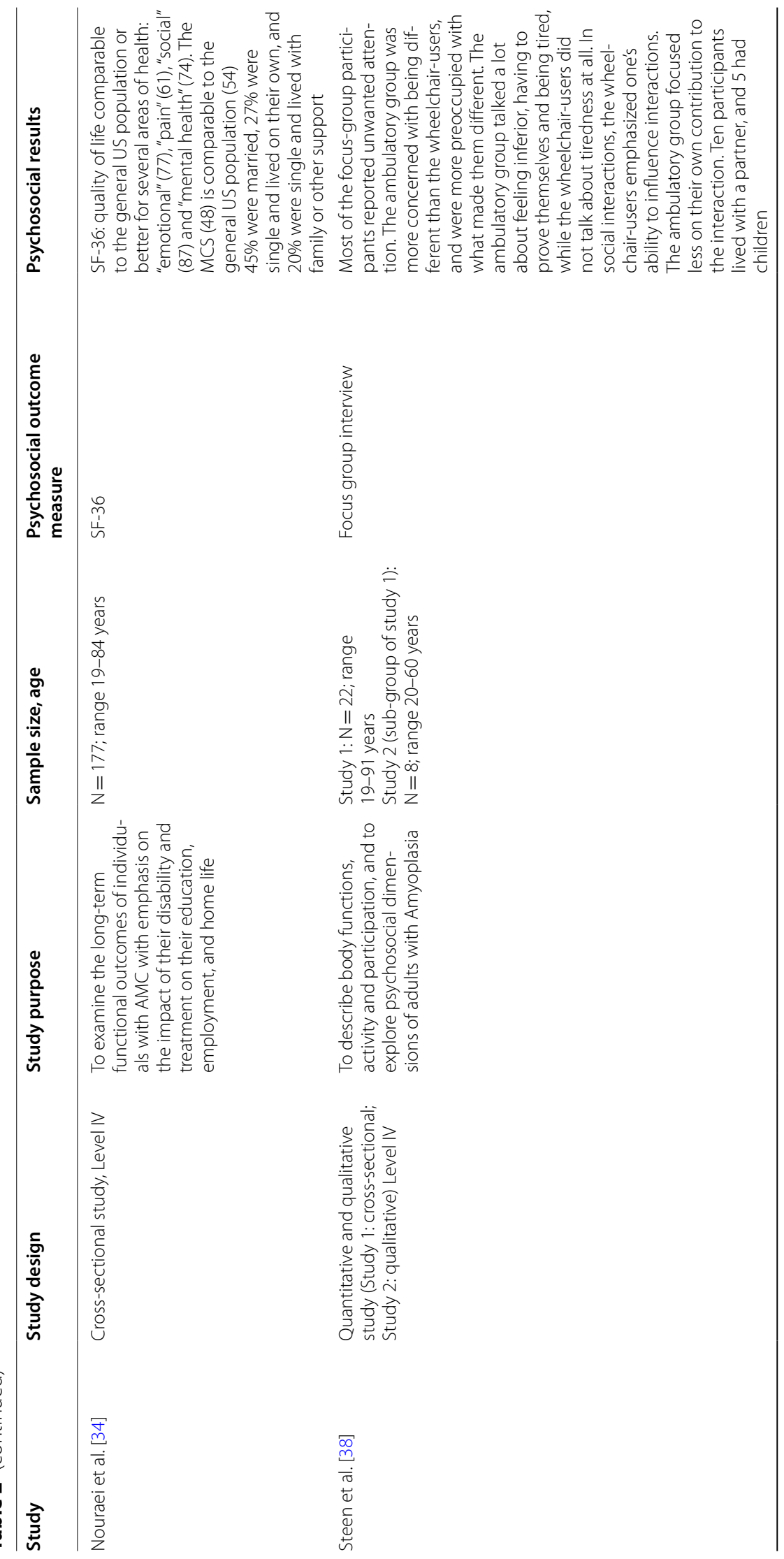




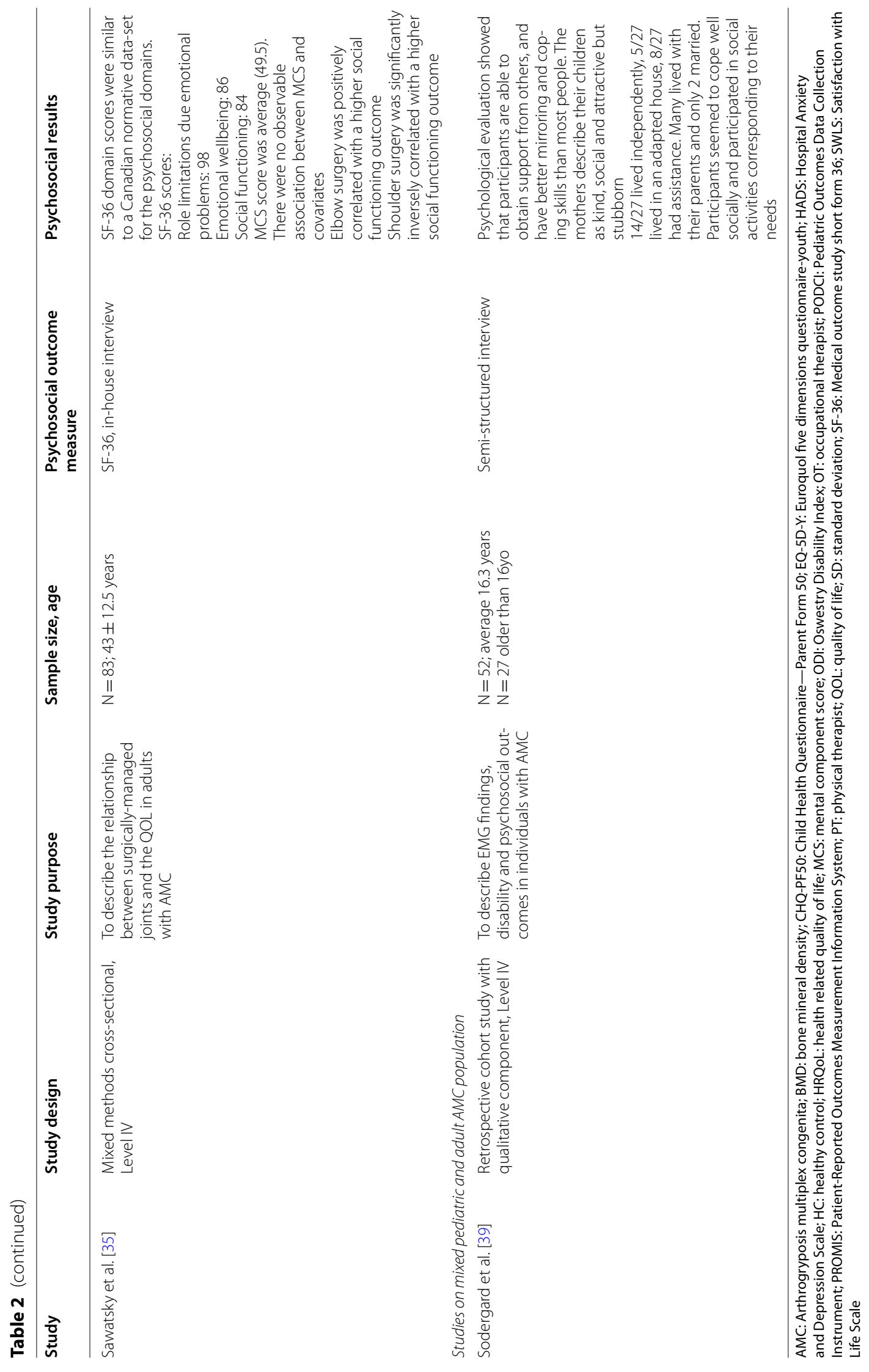


pertaining to psychosocial wellbeing included the Euroquol five dimensions questionnaire for youth (EQ5D-Y), and the Child Health Questionnaire-Parent Form (CHQ-PF50) [29]. Two studies used a semi-structured interview $[13,32]$.

The outcome measures used in the adult populations varied. Three studies used the Medical Outcome Study Short Form 36 (SF-36) [33-35]. Other outcomes measures used were the Satisfaction with Life Scale (SWLS) [38], the Hospital Anxiety and Depression Scales (HADS) [39], the Oswestry disability index (ODI), the EQ-5D [33], the PROMIS-57 v2.0 profile and PROMIS Global health profile [36]. One study used a focus group interview [40]. Two studies combined the use of a standardized outcome measures with a qualitative interview [33, 35]. The study with mixed pediatric and adult population used semi-structured interviews to evaluate psychosocial outcomes [39]. See Table 3 for a list of the outcomes measures used to evaluate psychosocial wellbeing in this population and their characteristics.

\section{Psychosocial outcomes in children with AMC}

Pediatric studies using the PODCI showed that "happiness" scores are generally high and comparable to the healthy population [5-7, 27-30]. These studies did not specifically aim to study psychosocial outcomes and most studies looked at outcomes of surgical interventions. One study comparing the effectiveness of the Ponseti method in treating idiopathic clubfoot and clubfoot associated with arthrogryposis showed that the arthrogryposis group had significantly lower "happiness" scores than the idiopathic group at follow-up, yet both groups reported high "happiness" scores [28]. Another study looking at long-term functional outcome of patients with arthrogryposis treated with surgical knee releases showed that the "happiness" normalized score was moderately positively correlated with the final average knee extension [6]. One study comparing functional and psychosocial outcomes between a group of children with Amyoplasia and a group of children with distal arthrogryposis showed no significant difference between the two groups in the "happiness" domain [30]. PROMIS scores of depression, anxiety, and peer relations domains were in the normal range for both groups but the "peer relations" score was statistically lower for the distal arthrogryposis group [30].

One study evaluating health related quality of life (HRQoL) in a group of children with AMC showed that, on the CHQ-50P, the AMC group scored lower than the healthy control on domains of parent impact/emotional, self-esteem and behavior which included satisfaction with school, athletic ability/outlook, looks/appearance, ability to get along with others and family, and life overall [31]. The same study compared children with AMC who wear orthoses to those who do not, and no significant differences were found between the two groups on the psychosocial outcome domains of the CHQ-50P and the EQ-5D.

In a qualitative study, youth reported, among other things, affective needs related to anxiety and body image and how those were impacted by factors such as pain and fatigue [13]. Parents reported the complexity of the needs to be taken into account when caring for a child with AMC, such as affective needs, physical needs, pain, mobility and adaptations, and how it affects the parent's ability to work [13].

Finally, based on results from qualitative interviews, a study showed the benefits of early assistive mobility using a toy car on social and communication development, motivation, and curiosity [32].

\section{Psychosocial outcomes in adults with AMC}

Three studies on adults with AMC used the SF-36 outcome measure and included 50 to 177 participants [3335]. Findings showed that the mental capacity score of individuals with $\mathrm{AMC}$, which encompasses the psychosocial domains of the measure, were comparable to the general USA and Canadian populations [33-35]. Separate scores for the psychosocial domains of the SF-36 were reported in two studies and were comparable to the general population [34, 35]. One of these studies described the relationship between surgically managed joints and the QOL in adults with AMC and showed that elbow surgery was positively correlated with a higher social functioning outcome and shoulder surgery was significantly inversely correlated with a higher social functioning outcome [35]. However, the strength of the correlation was not reported.

Altiok and colleagues reported that more than half of the adults with AMC in their study are satisfied to extremely satisfied with life [36]. The five participants who were dissatisfied with life also reported lower physical function, higher anxiety, depression and fatigue, and pain in multiple joints as per the PROMIS-57. Measures of global physical health, global mental health and pain level were moderately correlated with satisfaction with life. Scores of the PROMIS-57 and PROMIS global health psychosocial domains were consistent with the normative US population [36].

A study reported the presence of psychological problems associated with pain in less than half of the sample [37]. These problems were, in decreasing order of prevalence, anxiety, fatigue, difficulty with sexual life, altered self-esteem and feeling of solitude. They affected work and study in $17 \%$, and planning of parenthood in $7 \%$ of participants. Only one person was reported to present with some depression as per the HADS. There was no 


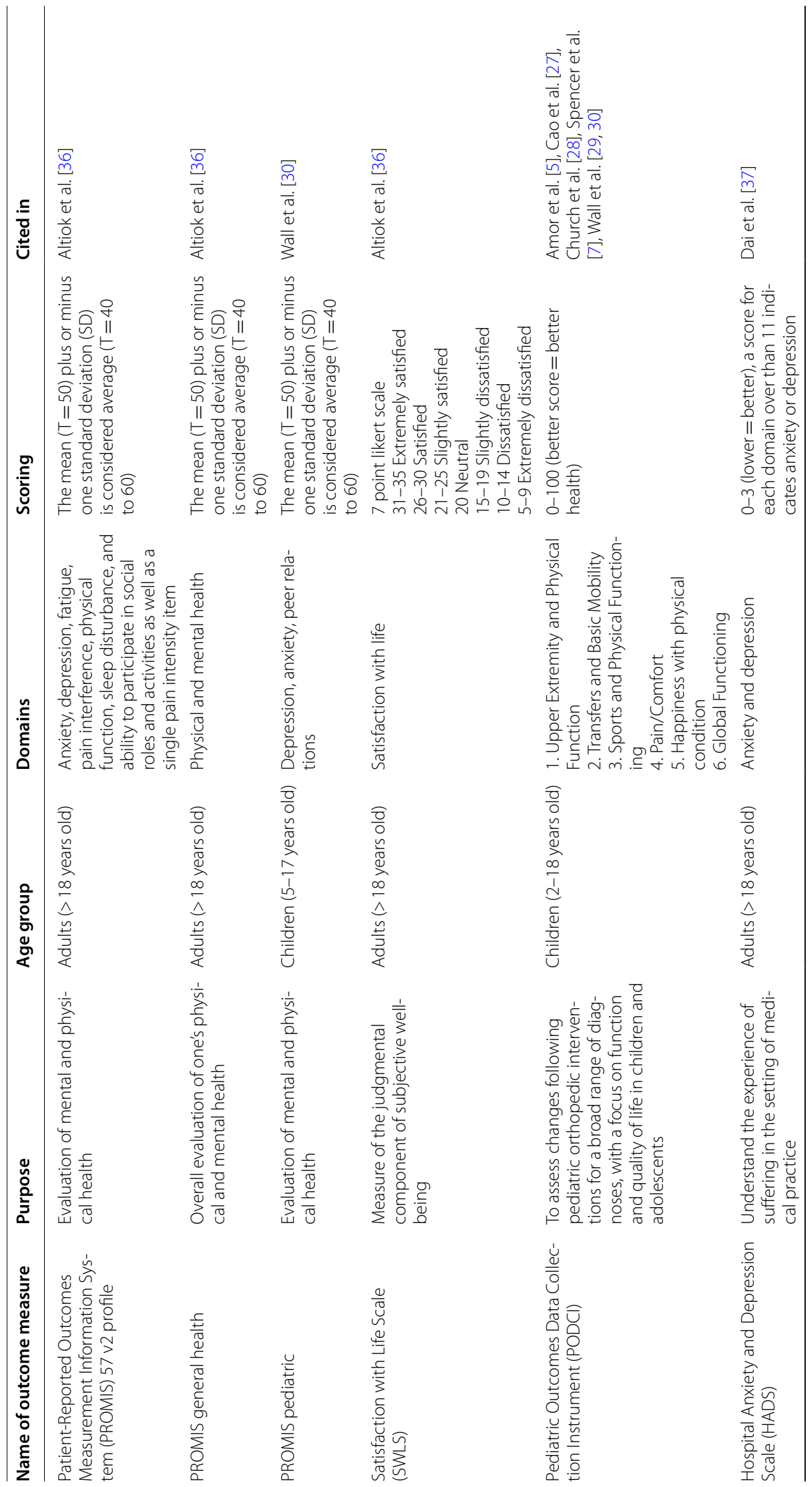




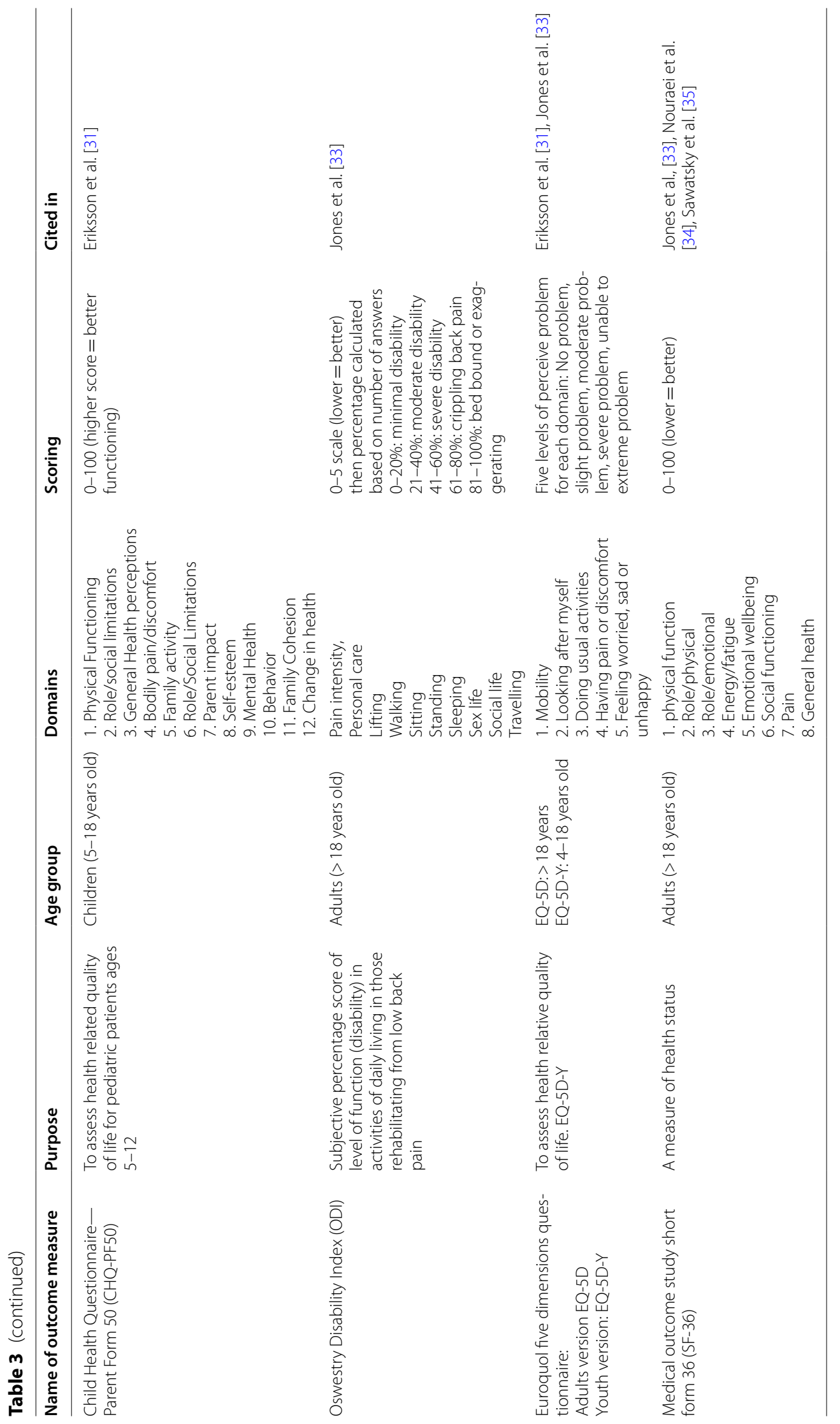


difference in the psychosocial outcomes between the Amyoplasia group and other types of AMC [37].

Another study looking at pain and psychosocial outcomes in adults with AMC showed low levels of disabling scores for pain and anxiety/depression on the EQ-5D and no pain interference in the ODI domains on social activities or sex life [33]. However, closed-ended questions showed that pain led to significant impairment in social activities and sex life.

A focus group conducted with eight adults with AMC showed the different perceptions of individuals who are ambulatory and those who are wheelchair users, and the differences in how they handle stigma in social situations [38]. Participants who were ambulatory were more concerned about their appearance and about looking different; they expressed feeling inferior and focused less on their contribution in social interactions. On the contrary, wheelchair users were less concerned about their differences and focused more on how they can contribute to social interactions [38].

\section{Other factors affecting psychosocial outcomes}

Five of the eight studies among adults with AMC reported data on the living situation and/or marital status of their participants [34, 36-39]. Two studies, one of which had a large sample size, reported a high number of individuals who were married or living with a partner $(45 \%)[34,38]$. Other studies reported a lower marriage/ partnership rate $(<13 \%)$ and had a younger sample [36, $37,39]$. Of those who were single, many lived with their family $[36,37,39]$. Positive relationships with family and friends, their support and involvement were reflected in three studies [32, 38, 39]. Support groups, mentioned in one study, were reported as beneficial as they provide the opportunity to meet, exchange and learn from others with similar conditions [38].

\section{Stakeholder consultation}

Three themes emerged during the conference calls held with the stakeholders: the indirect cost of having a child with AMC, the importance of sports and leisure participation in quality of life, and the importance of youth empowerment. More specifically, the group discussed the indirect cost of caring for a child with AMC (e.g., many medical appointments, missed work days, travel time to appointments) and the impact on families, such as level of stress, interpersonal relationships among family members and dynamics within the family unit. Participation in leisure and sports were described as having a positive impact on the mental wellbeing of individuals with AMC. Promoting participation in activities such as adapted sports were considered as one of the goals when treating or caring for a child with AMC. Finally, providing youth with sufficient information and decision-making power regarding the treatments they receive (e.g. surgery) may lead to better psychosocial outcomes and better acceptance of their condition in the adult age.

\section{Discussion}

The aim of this scoping review was to describe what is known about the psychosocial outcomes of children and adults with AMC. Overall, the mental health, emotional wellbeing and levels of happiness in children and adults with AMC was shown to be comparable to that of the general population.

\section{Psychosocial outcomes in children with AMC}

Most pediatric studies aimed to evaluate surgical outcomes or compare health outcomes of children with AMC with the general population. Although most of the studies did not specifically evaluate psychosocial outcomes, they reported the level of "happiness" in children with AMC to be high and comparable to the general population when measured with the PODCI [5-7, 27-30]. One study using the CHQ-PF50 reported that children with AMC scored lower than the control group on parent impact/emotional, self-esteem and behavior [31]. Clinically important aspects of a condition may not be captured in generic QOL measures. A disease-specific QOL measure may be more sensitive to detect change following treatment and may be advisable to develop for AMC [40]. In pediatrics, when outcome measures include selfreported and proxy versions, both should be completed when possible as parents' and children's perspectives may differ $[41,42]$.

Studies evaluating QOL showed that individuals with physical disabilities do not report lower QOL, satisfaction with life or poorer perceptions of their wellbeing [43]. A systematic review on QOL in children with osteogenesis imperfecta (OI) showed that psychosocial QOL in this population is similar to the general population [44]. In a qualitative study, children with OI expressed emotions of fear, pain, isolation and being different [45]. Similarly, the qualitative study by Elfassy and colleagues described the perception of youth with AMC with regards to pain, fatigue, anxiety and body image [13]. Qualitative studies can be useful for consideration, in complementarity to quantitative studies, to understand stakeholder lived experiences.

While the topic of bullying was not mentioned in the articles of this scoping review, it may be worth exploring as well. Children with disabilities may be at higher risk of bullying [46] as described in a qualitative study with children and youth with cerebral palsy (CP) [47]. Participation in leisure activities, discussed during consultation with stakeholders, contributes to the mental and physical 
wellbeing of children with physical disabilities [48, 49]. However, there is a lack of studies looking at leisure and participation in children with AMC [50]. A case report described the positive effects of hydrotherapy on a child with AMC [41]. Although in this context the activity was therapeutic, it is a glimpse of the potential positive impact of a fun inclusive activity on a child's self-esteem. The use of assistive technology, such as for mobility or self-care, may also facilitate social participation and interaction, and consequently improve self-esteem [32, 52, 53].

Family dynamics and challenges emerged as an important theme during the stakeholder consultation phase. A qualitative study described caregiver's perspective on managing their child's care and supporting their growth in different aspects of daily life [13]. These factors, and their impact on family dynamics, life balance and psychosocial wellbeing of the family as a unit should be considered in both research and in clinical practice.

\section{Psychosocial outcomes in adults with AMC}

Although we identified fewer studies among adults than children with AMC, a greater number of the studies among the adult population addressed psychosocial outcomes specifically, such as QOL, satisfaction with life and mental health. Overall, the psychosocial outcomes among adults with AMC were similar to the general population and levels of depression were low [33-37]. Pain and fatigue were important factors that had an impact on QOL, mental health, social relationships and intimacy, as well as family planning [33, 36, 37]. Therefore, it is important to offer this population a multidisciplinary approach to address elements of pain and to provide psychological follow-up and support to promote positive outcomes later in life. Two of the studies reporting on the marital and living situation of adults with AMC had a high number of participants who were married or living with a partner [34, 38], which is similar to findings from other studies on adults with AMC [54-56]. Other studies reported a lower number of married participants, which may be due to their sample being younger [36, 37, 39]. Support with family planning and obstetrical consultation are important to address concerns related to parenthood [57].

From childhood to adulthood, individuals with AMC undergo an average of four to nine surgeries [18, 34]. A study looking at the relationships between surgically managed joints and QOL in adults found correlations between certain upper limb surgeries and social functioning [35]. Longitudinal studies could help evaluate the impact of surgery on functional and psychosocial outcomes, and determine the types of surgeries that should be recommended for this population.

\section{Limitations}

Only French or English articles were included. Ongoing and non-published studies were not included in the search criteria. Stakeholders' input at the stakeholder consultation stage of this scoping review involved informal discussions on psychosocial wellbeing and may have been optimized by using a more formal approach with specific guiding questions.

\section{Conclusion}

Psychosocial outcomes among children and adults with AMC were found to be comparable to the general population. In adults, higher levels of pain and fatigue were associated with lower mental health and satisfaction with life and may have a negative impact on interpersonal relationships and intimacy. A large proportion of adults with AMC live with a partner or are married. The presence of meaningful relationships and access to support groups are valuable assets for this population. Psychosocial support should be part of the multidisciplinary management of AMC throughout the lifespan.

\begin{abstract}
Abbreviations
AMC: Arthrogryposis multiplex congenita; CP: Cerebral palsy; CHQ-PF50: Child Health Questionnaire-Parent Form 50; EQ-5D-Y: Euroquol five dimensions questionnaire-youth; HADS: Hospital Anxiety and Depression Scale; HRQoL: Health related quality of life; ODI: Oswestry Disability Index; Ol: Osteogenesis imperfecta; PODCl: Pediatric Outcomes Data Collection Instrument; PROMIS: Patient-Reported Outcomes Measurement Information System; QOL: Quality of life; SF-36: Medical outcome study short form 36; SWLS: Satisfaction with Life Scale.
\end{abstract}

\section{Supplementary Information}

The online version contains supplementary material available at https://doi. org/10.1186/s12955-021-01896-5.

Additional file 1. Ovid MEDLINE(R) ALL 1946 to March 06, 2020. Provides the search strategy used in Medline and applied to the other databases.

Acknowledgements

We would like to acknowledge Dr. André Bussières for his methodological guidance and advice.

\section{Authors' contributions}

SC and ND-O developed the research question and designed the project. They participated in developing the search strategy. They were the reviewers for the data selection. SC performed the data extraction and analysis, and drafted the manuscript. ND-O revised the manuscript. JB developed the search strategy and performed the literature searches in the different databases, and revised the manuscript. TW and FC contributed to the discussion points and the revision of the manuscript. All authors read and approved the final manuscript.

\section{Funding}

This scoping review was supported by a grant obtained by the Richard and Edith Strauss Canada Foundation (\#253636). Dr. Dahan-Oliel holds a clinician research scholar salary award from the Fonds de la Recherche en Santé du Québec. 


\section{Availability of data and materials}

Full data extraction table available upon request. Summary of results in Table 2.

\section{Declarations}

Ethics approval and consent to participate.

Not applicable.

\section{Consent for publication}

Not applicable.

\section{Competing interests}

The authors declare that they have no competing interests.

\section{Author details}

${ }^{1}$ Shriners Hospital for Children-Canada, Montreal, Canada. ${ }^{2}$ Schulich Library of Physical Sciences, Life Sciences, and Engineering, McGill University, Montreal, Canada. ${ }^{3}$ Orthotist-Prosthetist and Patient Partner, St-Zotique, Canada. ${ }^{4}$ Shriners Hospital for Children-Canada, School of Physical and Occupational Therapy, McGill University, Montreal, Canada.

\section{Received: 13 November 2020 Accepted: 18 November 2021}

Published online: 29 November 2021

\section{References}

1. Cachecho S, Elfassy C, Hamdy R, Rosenbaum P, Dahan-Oliel N. Arthrogryposis multiplex congenita definition: Update using an international consensus-based approach. Am J Med Genet C Semin Med Genet. 2019;181(3):280-7.

2. Fahy MJ, Hall JG. A retrospective study of pregnancy complications among 828 cases of arthrogryposis. Genet Couns. 1990;1(1):3-11.

3. Staheli L. Arthrogryposis: a text atlas. 1st ed. Cambridge: Cambridge University Press; 1998.

4. Kowalczyk B, Feluś J. Arthrogryposis: an update on clinical aspects, etiology, and treatment strategies. Arch Med Sci AMS. 2016;12(1):10.

5. Amor CJ, Spaeth MC, Chafey DH, Gogola GR. Use of the Pediatric Outcomes Data Collection Instrument to evaluate functional outcomes in arthrogryposis. J Pediatr Orthop. 2011;31(3):293-6.

6. Ho C, Karol K. The utility of knee releases in arthrogryposis. J Pediatric Orthop. 2008;28(3):307-13.

7. Spencer H, Bowen R, Caputo K, Green T, Lawrence J. Bone mineral density and functional measures in patients with arthrogryposis. J Pediatric Orthop. 2010;30(5):514-8.

8. Bender LH, Withrow CA. Arthrogryposis multiplex congenita. Orthop Nurs. 1989:8(5):29-35.

9. Bernstein RM. Arthrogryposis and amyoplasia. J Am Acad Orthop Surg. 2002;10(6):417-24.

10. Sells JM, Jaffe KM, Hall JG. Amyoplasia, the most common type of arthrogryposis: the potential for good outcome. Pediatrics. 1996;97(2):225-31.

11. Fassier A, Wicart P, Dubousset J, Seringe R. Arthrogryposis multiplex congenital. Long-term follow-up from birth until skeletal maturity. J Children's Orthop. 2009:3(5):383-90.

12. Hall JG, Agranovich O, Ponten E, van Bosse HJ. Summary of the 2nd international symposium on Arthrogryposis, St. Petersburg, Russia, Sptember 17-19, 2014. Am J Med Genet A. 2015;167(6):1193-5.

13. Elfassy C, Darsaklis B, Snider L, Gagnon C, Hamdy R, Dahan-Oliel N. Rehabilitation needs of youth with arthrogryposis multiplex congenita: perspectives from key stakeholders. Disabil Rehab. 2019;42:2318-24.

14. Townsend EA, Polatajko HJ. Advancing an occupational therapy vision for health, well-being, and justice through occupation. Ottawa: CAOT Publications ACE; 2007.

15. Diener E. Subjective well-being: the science of happiness and a proposal for a national index. Am Psychol. 2000;55(1):34

16. Ryff CD, Singer BH, Dienberg Love G. Positive health: connecting well-being with biology. Philos Trans R Soc Lond Ser B Biol Sci. 2004;359(1449):1383-94.
17. Voll R. Aspects of the quality of life of chronically ill and handicapped children and adolescents in outpatient and inpatient rehabilitation. Int J Rehabil Res. 2001;24(1):43-9.

18. Hansen D, Elfassy C, Montpetit K, Ghalimah B, Hamdy R, Dahan-Oliel N. A review of the orthopedic interventions and functional outcomes among a cohort of 114 children with arthrogryposis multiplex congenita. J Pediatr Rehab Med. 2020:13:263-71.

19. Cirillo A, Collins J, Sawatzky B, Hamdy R, Dahan-Oliel N. Pain among children and adults living with arthrogryposis multiplex congenita: a scoping review. Am J Med Genet C Semin Med Genet. 2019;181(3):436-45.

20. Thomas A, Lubarsky S, Durning SJ, Young ME. Knowledge syntheses in medical education: demystifying scoping reviews. Acad Med. 2017;92(2):161-6.

21. Arksey H, O'Malley L. Scoping studies: towards a methodological framework. Int J Soc Res Methodol. 2005;8(1):19-32.

22. Levac D. Scoping studies: advancing the methodology. Implement Sci. 2010;5:69.

23. Tricco AC, Lillie E, Zarin W, O'Brien KK, Colquhoun H, Levac D, Hempel S. PRISMA extension for scoping reviews (PRISMA-SCR): checklist and explanation. Ann Intern Med. 2018;169(7):467-73.

24. Ouzzani M, Hammady H, Fedorowicz Z, Elmagarmid A. Rayyan—a web and mobile app for systematic reviews. Syst Rev. 2016:5(1):210.

25. Wright JG, Swiontkowski MF, Heckman JD. Introducing levels of evidence to The Journal. J Bone Jt Surg. 2003;85(1):1.

26. Shippee ND, Domecq Garces JP, Prutsky Lopez GJ, Wang Z, Elraiyah TA, Nabhan M, Erwin PJ. Patient and service user engagement in research: a systematic review and synthesized framework. Health Expect. 2015;18(5):1151-66.

27. Cao J, Stutz C, Beckwith T, Browning A, Mills J, Oishi SN. Elbow release and tricepsplasty in arthrogrypotic patients: a long-term follow-up study. J Hand Surg. 2020:45(6):549-e1.

28. Church C, McGowan A, Henley J, Donohoe M, Niiler T, Shrader MW, Nichols LR. The 5-year outcome of the Ponseti method in children with idiopathic clubfoot and arthrogryposis. J Pediatr Orthop. 2020;40(7):e641-6.

29. Wall LB, Calhoun V, Roberts S, Goldfarb CA. Distal humerus external rotation osteotomy for hand position in arthrogryposis. J Hand Surg. 2017;42(6):473-e1.

30. Wall LB, Vuillerman C, Miller PE, Bae DS, Goldfarb CA, CoULD Study Group. Patient-reported outcomes in arthrogryposis. J Pediatr Orthop. 2020;40(7):357-60.

31. Eriksson M, Jylli L, Villard L, Kroksmark AK, Bartonek $\AA$. Health-related quality of life and orthosis use in a Swedish population with arthrogryposis. Prosthet Orthot Int. 2018;42(4):402-9.

32. Pritchard-Wiart L, Bragg E, Thompson-Hodgetts S. The young movers project: a case series describing modified toy car use as an early movement option for young children with mobility limitations. Phys Occup Ther Pediatr. 2019;39(6):598-613.

33. Jones T, Miller R, Street JT, Sawatzky B. Validation of the Oswestry Disability Index for pain and disability in arthrogryposis multiplex congenita. Ann Phys Rehabil Med. 2019;62(2):92-7.

34. Nouraei H, Sawatzky B, MacGillivray M, Hall J. Long-term functional and mobility outcomes for individuals with arthrogryposis multiplex congenita. Am J Med Genet A. 2017:173(5):1270-8.

35. Sawatzky B, Jones T, Miller R, Noureai $H$. The relationship between joint surgery and quality of life in adults with arthrogryposis: an international study. Am J Med Genet C Semin Med Genet. 2019;181(3):469-73.

36. Altiok H, Flanagan A, Krzak JJ, Hassani S. Quality of life, satisfaction with life, and functional mobility of young adults with arthrogryposis after leaving pediatric care. Am J Med Genet C Semin Med Genet. 2019:181(3):461-8.

37. Dai S, Dieterich $K$, Jaeger M, Wuyam B, Jouk PS, Pérennou D. Disability in adults with arthrogryposis is severe, partly invisible, and varies by genotype. Neurology. 2018;90(18):e1596-604.

38. Steen U, Christensen E, Samargian A. Adults living with amyoplasia: function, psychosocial aspects, and the benefit of AMC support groups. J Pediatr Orthop. 2017:37:S31-2.

39. Södergård J, Hakamies-Blomqvist L, Sainio K, Ryöppy S, Vuorinen R. Arthrogryposis multiplex congenita: perinatal and electromyographic findings, disability, and psychosocial outcome. J Pediatr Orthop B. 1997;6(3):167-71 
40. Eiser C. Children's quality of life measures. Arch Dis Child. 1997;77(4):350-4.

41. Varni JW, Burwinkle TM, Sherman SA, Hanna K, Berrin SJ, Malcarne VL, Chambers HG. Health-related quality of life of children and adolescents with cerebral palsy: hearing the voices of the children. Dev Med Child Neurol. 2005;47(9):592-7.

42. Eiser C, Morse R. Can parents rate their child's health-related quality of life? Results of a systematic review. Qual Life Res. 2001;10(4):347-57.

43. Rosenbaum PL, Livingston MH, Palisano RJ, Galuppi BE, Russell DJ. Quality of life and health-related quality of life of adolescents with cerebral palsy. Dev Med Child Neurol. 2007:49(7):516-21.

44. Dahan-Oliel N, Oliel S, Tsimicalis A, Montpetit K, Rauch F, Dogba MJ. Quality of life in osteogenesis imperfecta: a mixed-methods systematic review. Am J Med Genet A. 2016;170(1):62-76.

45. Hill CL, Baird WO, Walters SJ. Quality of life in children and adolescents with Osteogenesis Imperfecta: a qualitative interview based study. Health Qual Life Outcomes. 2014;12(1):1-9.

46. Cummings JG, Pepler DJ, Mishna F, Craig WM. Bullying and victimization among students with exceptionalities. Except Educ Can. 2006;16(3):193-222.

47. Lindsay S, McPherson AC. Experiences of social exclusion and bullying at school among children and youth with cerebral palsy. Disabil Rehabil. 2012;34(2):101-9.

48. Dahan-Oliel N, Shikako-Thomas K, Majnemer A. Quality of life and leisure participation in children with neurodevelopmental disabilities: a thematic analysis of the literature. Qual Life Res. 2012;21(3):427-39.

49. Shikako-Thomas K, Dahan-Oliel N, Shevell M, Law M, Birnbaum R, Rosenbaum P, Majnemer A. Play and be happy? Leisure participation and quality of life in school-aged children with cerebral palsy. Int J Pediatr. 2012;2012:387280.

50. Elfassy C, Cachecho S, Snider L, Dahan-Oliel N. Participation among children with arthrogryposis multiplex congenita: a scoping review. Phys Occup Therapy Pediatr. 2020;40:1-28.

51. Campbell C. Hydrotherapy: a way for exceptional children to swim toward success. A young boy with special needs learns confidence and independence through a special swimming program. Except Parent. 2005;35(6):26

52. Dalton C, Hoyt-Hallett G. Enablement through provision of assistive technology: case reports of two children with physical disabilities. Br J Occup Ther. 2013;76(2):108-11.

53. Haumont T, Rahman T, Sample W, King MM, Church C, Henley J, Jayakumar S. Wilmington robotic exoskeleton: a novel device to maintain arm improvement in muscular disease. J Pediatr Orthop. 2011;31(5):e44-9.

54. Carlson WO, Speck GJ, Vicari V, Wenger DR. Arthrogryposis multiplex congenita: a long-term follow-up study. Clin Orthop Relat Res. 1985:194:115-24.

55. Dubousset J, Guillaumat M. Long-term outcome for patients with arthrogryposis multiplex congenita. J Child Orthop. 2015;9(6):449-58.

56. Steen $U$, Wekre LL, Vøllestad NK. Physical functioning and activities of daily living in adults with amyoplasia, the most common form of arthrogryposis. A cross-sectional study. Disabil Rehabil. 2018;40(23):2767-79.

57. Castro J, Abreu-Silva J, Godinho C, Valente F. Successful pregnancy in a woman with arthrogryposis multiplex congenita. Case Rep. 2013;2013:bcr2013201621.

\section{Publisher's Note}

Springer Nature remains neutral with regard to jurisdictional claims in published maps and institutional affiliations.

Ready to submit your research? Choose BMC and benefit from:

- fast, convenient online submission

- thorough peer review by experienced researchers in your field

- rapid publication on acceptance

- support for research data, including large and complex data types

- gold Open Access which fosters wider collaboration and increased citations

- maximum visibility for your research: over $100 \mathrm{M}$ website views per year

At BMC, research is always in progress.

Learn more biomedcentral.com/submissions 